\title{
Bosonic entanglement renormalization circuits from wavelet theory
}

\author{
F. G. Witteveen ${ }^{1, *}$, M. Walter ${ }^{1,2}$ \\ 1 Korteweg-de Vries Institute for Mathematics and QuSoft, University of Amsterdam, the \\ Netherlands \\ 2 Institute for Theoretical Physics, Institute for Language, Logic, and Computation, \\ University of Amsterdam, the Netherlands \\ * f.g.witteveen@uva.nl
}

September 1, 2021

\begin{abstract}
Entanglement renormalization is a unitary real-space renormalization scheme. The corresponding quantum circuits or tensor networks are known as MERA, and they are particularly well-suited to describing quantum systems at criticality. In this work we show how to construct Gaussian bosonic quantum circuits that implement entanglement renormalization for ground states of arbitrary free bosonic chains. The construction is based on wavelet theory, and the dispersion relation of the Hamiltonian is translated into a filter design problem. We give a general algorithm that approximately solves this design problem and prove an approximation result that relates the properties of the filters to the accuracy of the corresponding quantum circuits. Finally, we explain how the continuum limit (a free bosonic quantum field) emerges naturally from the wavelet construction.
\end{abstract}

\section{Contents}

1 Introduction $\quad 2$

2 Perfect reconstruction and biorthogonal filters 3

3 Entanglement renormalization and filter design 4

3.1 Derivation of filter condition 6

$\begin{array}{lll}4 & \text { Entanglement renormalization circuits } & 7\end{array}$

5 The continuum limit $\quad 8$

5.1 Scaling and wavelet functions 9

5.2 Entanglement renormalization for the massless boson 9

5.3 The massive bosonic field 10

5.4 Other perspectives 11

6 Conclusion 11 
A Construction of filters

B Construction of circuits from filters

C Approximation theorem $\quad 16$

References $\quad 21$

\section{Introduction}

Real-space renormalization methods are a powerful tool to study strongly interacting quantum systems. In one spatial dimensional, prominent examples are the Density Matrix Matrix Renormalization Group [1], with the associated tensor network class of Matrix Product States (MPS) [2] and entanglement renormalization [3], with the corresponding Multiscale Entanglement Renormalization Ansatz (MERA) tensor network states [3]. Entanglement renormalization implements a real-space renormalization by a local unitary transformation, decomposing a state into a product state and the renormalized state. By applying many such layers one can build a highly entangled state from product states, as in Fig. 1. Scale-invariant MERA states are a good variantional class for approximating ground states of critical quantum chains and one can extract the conformal data of the continuum limit conformal field theory of the system from the entanglement renormalization superoperator [4]. If the entanglement renormalization unitaries are implemented by low-depth local quantum circuits we will call this an entanglement renormalization circuit. This class of states can be prepared very efficiently on a quantum computer, providing a promising candidate for simulation of quantum systems on a quantum computer [5. Entanglement renormalization circuits are very efficient compared to other methods [6 as they are logarithmic in the system size, and the single layer circuit depth needed for a given error typically scales polylogarithmically in the error, and can deal with gapless systems.

Unfortunately, compared to for instance Matrix Product States (MPS), our analytic understanding of MERA is limited. One direction in which progress to analytic understanding has been made is in connection to wavelets. It has been observed $[7,8$ that the fermionic second quantization of a discrete wavelet transform gives rise to a MERA, and that its continuum limit can be related to the corresponding wavelet functions [9]. In [7] it was suggested that a similar result could also be true for free bosonic systems, for which one can also define entanglement renormalization in the Gaussian, or linear optics, formalism [10].

In this work we explain how one can indeed construct a Gaussian bosonic entanglement renormalization scheme for bosonic quadratic one-dimensional Hamiltonians, using the second quantization of biorthogonal wavelet filters or perfect reconstruction filters. The resulting entanglement renormalization takes the form of a short depth Gaussian bosonic circuit, providing evidence for the relevance of entanglement renormalization circuits for preparing ground states of (near) critical quantum systems. Moreover we can relate, similar as in the fermionic case [8], properties of the biorthogonal wavelet transform to the resulting MERA state, and we prove a rigorous approximation theorem for the correlation functions of the 


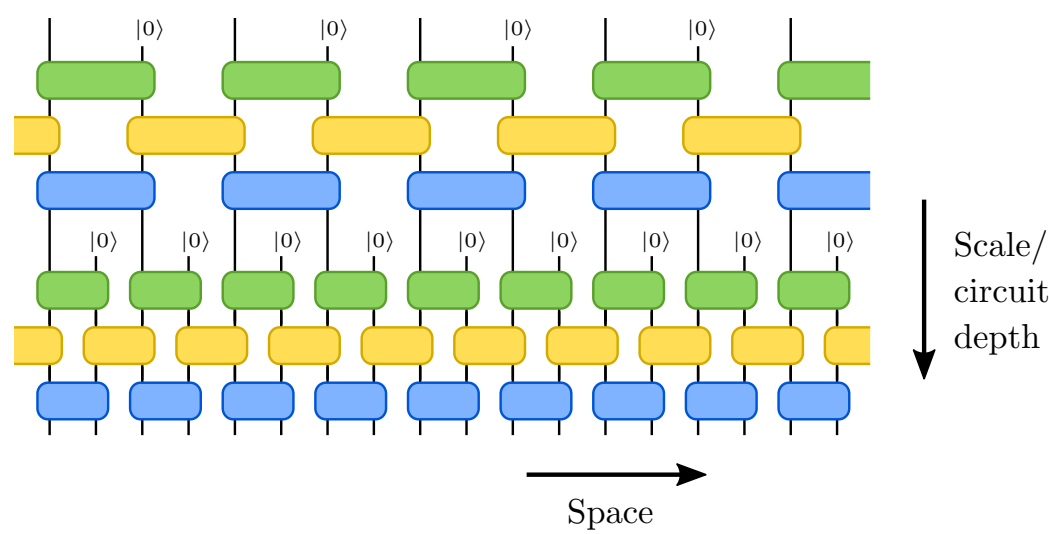

Figure 1: The structure of an entanglement renormalization circuit. Each layer is a constant depth quantum circuit that is supposed to implement a real-space renormalization. Every layer takes as input the output of the previous layer and a product state, resulting in an (entangled) MERA state at the bottom. Deeper layers correspond to structure at larger scales.

MERA state. Interestingly, our formalism is not restricted to the scale-invariant case, but can be used to construct entanglement renormalization circuits for arbitrary translation invariant quadratic bosonic Hamiltonians. Given such a Hamiltonian, we explain how a corresponding (approximate) entanglement renormalization circuit can be found by solving a filter design problem. We also give a general framework for constructing such filters, similar to the construction of the Daubechies wavelets. Finally, the continuum limit of the discrete system is directly related to the biorthogonal scaling and wavelet functions corresponding to the filters. For the free massless boson our construction reproduces various scaling dimensions exactly. If the system is not scale-invariant, we explain how one can still define versions of the wavelet and scaling functions which are not scale-invariant.

A natural application of a quantum computer based on bosonic variables [11] is to simulate bosonic quantum field theories [12] and wavelets are a very efficient choice of basis to discretize a quantum field theory for this purpose [13]. We explain that for any free 1+1-dimensional bosonic field theory, one can use suitably chosen biorthogonal wavelets to discretize the theory and use the corresponding wavelet decomposition to prepare its (approximate) ground state using a bosonic Gaussian quantum circuit. This provides a potential starting point to efficiently simulate interacting field theories on quantum computers.

\section{Perfect reconstruction and biorthogonal filters}

Perfect reconstruction filters, or biorthogonal wavelet filters, are filters that decompose a signal into a high-frequency part and a low frequency part. This is reminiscent of the disentangling procedure of entanglement renormalization, and in this work we explain the precise connection. Here we give a brief account of the theory of biorthogonal wavelet filters, see [14] for an introduction. We consider a pair of real-valued filters $g_{s}, h_{s} \in \ell^{2}(\mathbb{Z})$, called the scaling or low-pass filters. Often they will be finite impulse response (FIR) filters, meaning that they have finite support. We demand that these filters satisfy the perfect reconstruction condition on their Fourier transforms

$$
g_{s}(k) \overline{h_{s}(k)}+g_{s}(k+\pi) \overline{h_{s}(k+\pi)}=2
$$




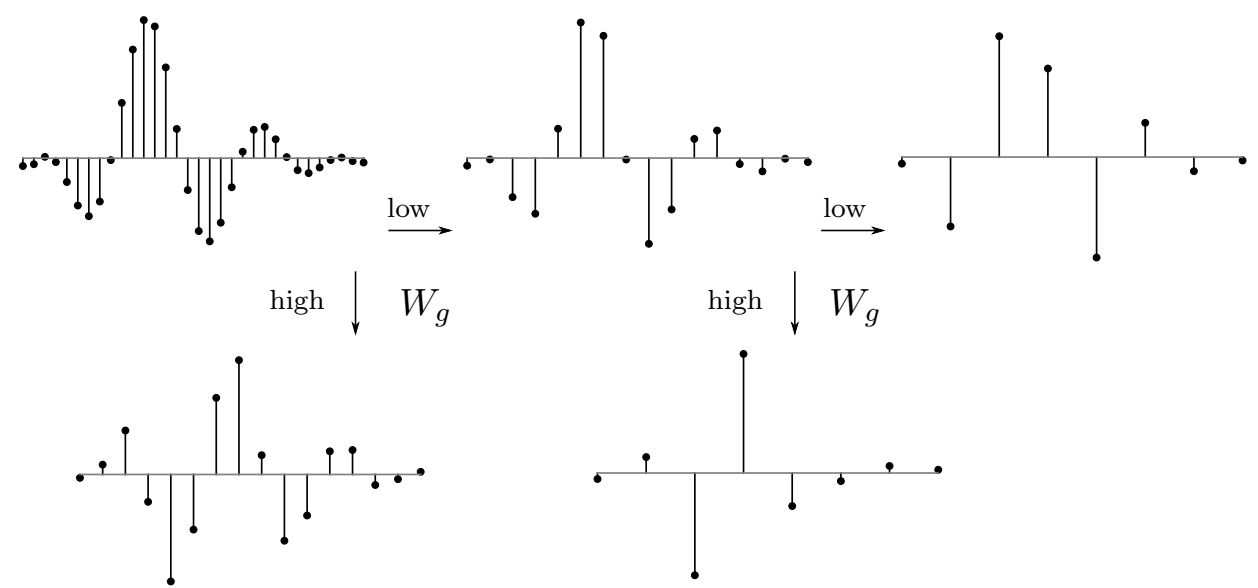

Figure 2: Iterating the wavelet decomposition $W_{g}$ resolves a signal into scales. Illustration for the Haar wavelet filters [9].

and define corresponding wavelet or high-pass filters by $g_{w}(k)=e^{-i k} \overline{h_{s}(k+\pi)}, h_{w}(k)=$ $e^{-i k} \overline{g_{s}(k+\pi)}$. These filters can be used to separate a signal $\{f[n]\}_{n \in \mathbb{Z}}$ into a low-frequency and a high frequency component, and conversely to reconstruct the original signal from these components. For this, we let $W_{g} f=f^{\text {low }} \oplus f^{\text {high }}$, where

$$
\begin{aligned}
f^{\text {low }}[n] & =\sum_{l} g_{s}[l] f[2 n+l], \\
f^{\text {high }}[n] & =\sum_{l} g_{w}[l] f[2 n+l]
\end{aligned}
$$

and we similarly define $W_{h}$. By applying this decomposition again to $f^{\text {low }}$, the original signal is recursively resolved into scales, see Fig. 2, It follows from Eq. (1) that $f$ can be reconstructed from its decomposition $W_{g} f$ by applying the transposed operation $W_{h}^{\top}$, so $W_{g}^{-1}=W_{h}^{\top}$ and

$$
f[n]=\sum_{l} h_{s}[n-2 l] f^{\text {low }}[l]+h_{w}[n-2 l] f^{\text {high }}[l] .
$$

The roles of $g$ and $h$ can be exchanged in this procedure.

\section{Entanglement renormalization and filter design}

We will consider translation invariant chains of harmonic oscillators $\left(q_{n}, p_{n}\right)$, with a Hamiltonian of the form

$$
H=\frac{1}{2}\left(\sum_{n \in \mathbb{Z}} p_{n}^{2}+\sum_{n, m \in \mathbb{Z}} q_{n} V_{n-m} q_{m}\right),
$$

where $V_{n m}=V_{n-m}$ defines a positive definite symmetric matrix. The ground state of such a quadratic Hamiltonian is a Gaussian state, determined by the dispersion relation $\omega(k)$ of the Hamiltonian. We study Gaussian circuits that map an unentangled state to the entangled ground state of a translation invariant Hamiltonian (or conversely, disentangle the ground state to an unentangled state). We consider Gaussian maps defined by $\tilde{q}_{n}=\sum_{m} A_{n m} q_{m}$, 
$\tilde{p}_{n}=\sum_{m} B_{n m} p_{m}$. This preserves the canonical commutation relations if and only if the matrices $A$ and $B$ are such that $B=\left(A^{\top}\right)^{-1}$. By a Gaussian circuit we will hence understand a sequence of Gaussian maps, each of which maps modes $\left(q_{n}, p_{n}\right)$ to a linear combination of itself and its direct neighbours. For details about quadratic bosonic Hamiltonians and Gaussian states see, for instance, 11, 15, 17. In Fourier space one simply maps a product state to the state with dispersion relation $\omega(k)$ by appropriately 'squeezing' each Fourier mode. However, this is a very non-local operation, whereas we are interested in a procedure that is local in real space. For more discussion of this point and variational algorithms to find Gaussian entanglement renormalization maps, see 10.

Since $W_{g}^{-1}=W_{h}^{\top}$ the map $W=W_{g} \oplus W_{h}$ defines a Gaussian map for any pair of biorthogonal wavelet filters $(g, h)$. This has the structure of a layer of entanglement renormalization, filtering out the high frequency modes. However, we need to choose the filters $g$ and $h$ such that $W$ actually disentangles the state, and the wavelet output is unentangled. If we normalize the dispersion relation such that $\omega(\pi)=1$, then the condition for the wavelet output to be disentangled is that the Fourier transforms of the filters satisfy

$$
g_{w}(k)=\omega(k) h_{w}(k) .
$$

Intuitively, what happens is that $W$ separates the bosonic modes in high frequency and low frequency modes, and Eq. (3) makes sure that the high frequency modes are not entangled to the low frequency modes in the ground state. We derive this condition below in Section 3.1 . The scaling (low frequency) modes are again mapped to a Gaussian state, possibly with a different dispersion relation. We can now recursively apply the same construction to the scaling output, as in Fig. 1, now with the renormalized dispersion relation, given by

$$
\omega(k) \mapsto \omega\left(\frac{k}{2}\right) \omega\left(\frac{k}{2}+\pi\right)
$$

and applying a 'squeezing' normalization layer to ensure the normalization $\omega(\pi)=1$.

While we motivated the procedure from the perspective of disentangling a given entangled state, the resulting circuit can also be used in the opposite direction, to prepare the ground state by applying the circuit to a product state, thus realizing the state as a bosonic MERA state. A paradigmatic example is the harmonic chain,

$$
H=\frac{1}{2}\left(\sum_{n \in \mathbb{Z}} p_{n}^{2}+m^{2} q_{n}^{2}+\frac{1}{4}\left(q_{n}-q_{n+1}\right)^{2}\right),
$$

which has dispersion relation $\omega(k)=\sqrt{m^{2}+\sin ^{2}\left(\frac{k}{2}\right)}$. In particular the massless harmonic chain is gapless and has dispersion relation $\omega(k)=\left|\sin \left(\frac{k}{2}\right)\right|$. It has the property that the dispersion relation is invariant under the renormalization step in Eq. (4). Hence the state on the scaling output of the entanglement renormalization will be the same after any number of layers. This implies that we can keep iterating the same entanglement renormalization layer with identical filters at each layer, giving a scale-invariant bosonic entanglement renormalization procedure for the massless harmonic chain.

In the massive case, the mass renormalizes as

$$
m \mapsto 2 \sqrt{m^{2}+m^{4}} .
$$

This is a relevant perturbation to the massless chain [10], and with increasing $l$ the dispersion relation becomes flat; correspondingly we can let the filters at the deeper layers approach orthogonal wavelet filters. 


\subsection{Derivation of filter condition}

We will now derive Eq. (3). The ground state of the Hamiltonian in Eq. (2) is completely determined by its covariance matrix $\gamma=\gamma^{q} \oplus \gamma^{p}$, whose Fourier transform is given by

$$
\begin{aligned}
\gamma^{q}(k) & =\frac{1}{2 \omega(k)} \\
\gamma^{p}(k) & =\frac{\omega(k)}{2} .
\end{aligned}
$$

The covariance matrix of an unentangled (uncorrelated) product state is $\frac{1}{2} \mathbb{1}$. Recall that any symplectic linear map $S$ on the set of modes $\left(q_{n}, p_{n}\right)$ defines a unitary map which maps Gaussian states to Gaussian states. Under a map of the form $A \oplus\left(A^{\top}\right)^{-1}$ the covariance matrix transforms as

$$
\begin{aligned}
\gamma^{q} & \mapsto A \gamma^{q} A^{\top} \\
\gamma^{p} & \mapsto\left(A^{\top}\right)^{-1} \gamma^{p} A^{-1} .
\end{aligned}
$$

We first normalize such that $\omega(\pi)=1$, which can be implemented by the symplectic (squeezing) map $(\sqrt{\omega(\pi)} \mathbb{1}) \oplus(1 / \sqrt{\omega(\pi)} \mathbb{1})$. Suppose we have filters $(g, h)$ satisfying Eq. (3), then $W=W_{g} \oplus W_{h}$ disentangles the ground state. To see that this is indeed true, we compute the result of applying the wavelet decomposition map to the ground state covariance matrix $\gamma=\gamma^{q} \oplus \gamma^{p}$ given in terms of the dispersion relation by Eq. (7). For this, we remark that from $g_{w}(k)=\omega(k) h_{w}(k)$ it follows that $h_{s}(k)=\omega(k+\pi) g_{s}(k)$. Then,

$$
\begin{aligned}
\omega(k) h_{w}(k) f^{\text {high }}(2 k) & =g_{w}(k) f^{\text {high }}(2 k), \\
\omega(k) h_{s}(k) f^{\text {low }}(2 k) & =g_{s}(k) \omega^{(1)}(2 k) f^{\text {low }}(2 k),
\end{aligned}
$$

where $\omega^{(1)}$ is the renormalized dispersion relation on the scaling output defined in Eq. (4) in the main text. This shows that $\omega(k) W_{h}^{\top}=W_{g}^{\top}\left(\omega^{(1)} \oplus \mathbb{1}\right)$ and hence

$$
\begin{aligned}
W_{h} \gamma^{p} W_{h}^{\top} & =W_{h} W_{g}^{\top}\left(\gamma^{p,(1)} \oplus \frac{1}{2} \mathbb{1}\right)=\gamma^{p,(1)} \oplus \frac{1}{2} \mathbb{1} \\
\gamma^{p,(1)}(k) & =\frac{1}{2} \omega^{(1)}(k) .
\end{aligned}
$$

Similarly, it holds that

$$
\begin{aligned}
W_{g} \gamma^{q} W_{g}^{\top} & =\gamma^{q,(1)} \oplus \frac{1}{2} \mathbb{1} \\
\gamma^{q,(1)}(k) & =\frac{1}{2 \omega^{(1)}(k)} .
\end{aligned}
$$

We thus see that $W$ has unentangled the high-frequency modes to a product state, and the low frequency modes are renormalized to have a new dispersion relation $\omega^{(1)}$ given by Eq. (4).

The full entanglement renormalization circuit consists of repeated applications of such layers. To introduce some notation, we let $\omega^{(l)}$ be the dispersion relation after $l$ layers of renormalization, recursively defined by (cf. Eq. (4))

$$
\omega^{(l+1)}(k)=\frac{\omega^{(l)}\left(\frac{k}{2}\right)}{\omega^{(l)}(\pi)} \frac{\omega^{(l)}\left(\frac{k}{2}+\pi\right)}{\omega^{(l)}(\pi)} .
$$


The normalization by $\omega^{(l)}(\pi)$ could also be absorbed in the filters, but we would like the filters to be such that $g(0)=h(0)=\sqrt{2}$, as is standard in the signal processing literature and convenient for the analysis. Then at the $l$-th layer we need filters $g^{(l)}, h^{(l)}$ satisfying $g_{w}^{(l)}(k)=\frac{\omega^{(l)}(k)}{\omega^{(l)}(\pi)} h_{w}^{(l)}(k)($ cf. Eq. 33$)$, and we let

$$
\begin{aligned}
& R_{g^{(l)}}=W_{g^{(l)}} \sqrt{\omega^{(l)}(\pi)}, \\
& R_{h^{(l)}}=W_{h^{(l)}} \frac{1}{\sqrt{\omega^{(l)}(\pi)}} .
\end{aligned}
$$

Finally, we define the $\mathcal{L}$-layer renormalization map as $R^{(\mathcal{L})}=R_{g}^{(\mathcal{L})} \oplus R_{h}^{(\mathcal{L})}$, where $R_{a}^{(\mathcal{L})}=$ $\left(R_{a(\mathcal{L}-1)} \oplus \mathbb{1}^{\oplus(\mathcal{L}-1)}\right) \circ \ldots \circ\left(R_{a^{(1)}} \oplus \mathbb{1}\right) \circ R_{a^{(0)}}$ for $a=g, h$. Then, $R^{(\mathcal{L})}$ maps the state with dispersion relation $\omega$ to a product state with covariance matrix $\frac{1}{2} \mathbb{1}$ on the $\mathcal{L}$ high frequency levels, and a state with dispersion relation $\omega^{(\mathcal{L})}$ on the remaining low frequency level.

\section{Entanglement renormalization circuits}

If $g$ and $h$ are FIR filters of size $2 M$, we show in Appendix B that $W$ gives rise to a Gaussian circuit of depth $M$ that maps the low-frequency modes to the odd sublattice and the highfrequency modes to the even sublattice as shown in Fig. 3. This is exactly the structure of an entanglement renormalization circuit. The converse to this construction is also true: any Gaussian entanglement renormalization circuit as described above arises in this way.

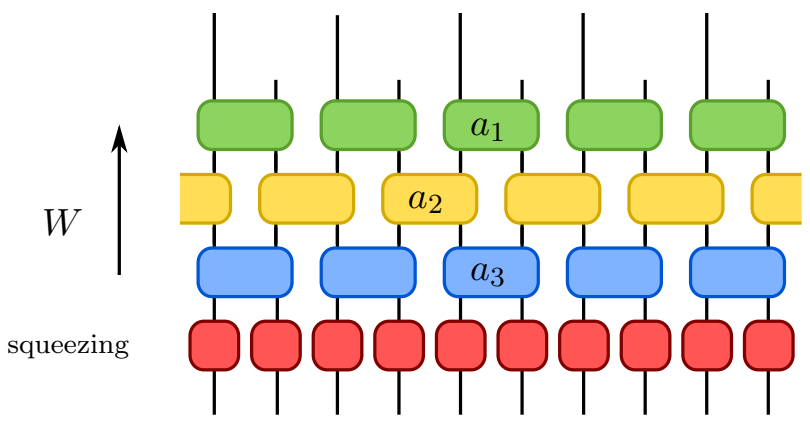

Figure 3: Decomposition of the entanglement renormalization map $R$ as a circuit. The wavelet transform $W=W_{g} \oplus W_{h}$ is decomposed as a Gaussian circuit with two-local gates $a_{i}$, and follows the bottom layer which squeezes by $\omega(\pi)^{\frac{1}{2}} \oplus \omega(\pi)^{-\frac{1}{2}}$ to normalize the dispersion relation.

When using a finite depth circuit, we may not be able to satisfy the relation in Eq. (3) exactly if $\omega(k)$ is not a ratio of trigonometric polynomials. In particular, this is the case for the harmonic chain. In this case we can still hope to approximate the dispersion relation, and correspondingly prepare a state that is close the true ground state. This raises two interesting questions. Firstly, the existence of filters that approximately satisfy Eq. (3) is not clear. In Appendix A we describe an explicit procedure for constructing such filters. Secondly, one can wonder whether a good approximation of the dispersion relation at the level of a single layer will indeed give rise to a good approximation of the ground state. Fortunately, the structure of entanglement renormalization is remarkably robust to small errors [5, 18], and 
in 8 a robustness result for wavelet based fermionic entanglement renormalization was proven. The bosonic setting is somewhat different, as the Hilbert spaces are infinite dimensional. We will now discuss that when the family of filters has a well-defined 'continuum limit', we can nevertheless prove a rigorous approximation theorem.

Suppose we are given a family of filter pairs $\left(g^{(l)}, h^{(l)}\right)$, where the $l$-th pair represents the $l$-th layer such that

$$
\left|g_{w}^{(l)}(k)-\frac{\omega^{(l)}(k)}{\omega^{(l)}(\pi)} h_{w}^{(l)}(k)\right| \leq \varepsilon,
$$

so they approximately reproduce the dispersion relation at each layer (up to normalization). Moreover, we need these families of filters to give rise to a 'stable' wavelet decomposition, in the sense that many iterations of the decomposition maps yield a uniformly bounded map. This is a standard assumption in wavelet theory. If we are only interested in an approximation, and the theory flows to either a critical theory or a trivial theory we only need a small number of 'transition layers' and pick fixed filters $\left(g^{(l)}, h^{(l)}\right)=(g, h)$ for large $l$. In Appendix C, we prove a general approximation theorem in this setting, which applies to an arbitrary quadratic Hamiltonian. We may measure the error in the two-point functions $\left\langle p_{i} p_{j}\right\rangle$ and $\left\langle q_{i} q_{j}\right\rangle$ (or covariance matrix). In the particular case of the harmonic chain in Eq. (5), our result specializes as follows.

Theorem (Informal). For the harmonic chain with mass $m$, the approximation error is bounded by

$$
\begin{aligned}
&\left|\left\langle p_{i} p_{j}\right\rangle-\left\langle p_{i} p_{j}\right\rangle_{\mathrm{MERA}}\right| \leq\left(\mathcal{O}\left(2^{-\frac{\mathcal{L}}{2}}\right)+\mathcal{O}\left(\varepsilon \log \frac{1}{\varepsilon}\right)\right) \sqrt{m^{2}+1} \\
&\left|\left\langle q_{i} q_{j}\right\rangle-\left\langle q_{i} q_{j}\right\rangle_{\mathrm{MERA}}\right| \leq\left(\mathcal{O}\left(2^{-\frac{\mathcal{L}}{2}}\right)+\mathcal{O}\left(\varepsilon \log \frac{1}{\varepsilon}\right)\right) \frac{1}{m}
\end{aligned}
$$

In the massless case, the latter bound is replaced by

$$
\left|\left\langle q_{i} q_{j}\right\rangle-\left\langle q_{i} q_{j}\right\rangle_{\mathrm{MERA}}\right| \leq\left(O\left(2^{-\frac{\mathcal{L}}{2}}\right)+\mathcal{O}\left(\varepsilon \log \frac{1}{\varepsilon}\right)\right) \sqrt{|i-j|} .
$$

Notice that in the massless case, there is an IR divergence, and $\left\langle q_{i} q_{j}\right\rangle$ is only defined up to a constant factor. The intuition behind the proof is that the contribution of the $\mathcal{L}$-th layer to the correlation function is bounded by $\mathcal{O}\left(2^{-\frac{\mathcal{L}}{2}}\right)$, so we need $\mathcal{O}\left(\log \frac{1}{\delta}\right)$ layers to get within error $\delta$ (even with perfect filters), while each layer contributes a factor of $\varepsilon$ to the error in the filter relation. Balancing these two contributions yields the desired bound.

If we denote by $M$ the circuit depth of a single layer, then we find numerically that $\varepsilon$ is exponentially small as a function of $M$, whereas the other wavelet-dependent parameters we have suppressed above only grow polynomially. Hence, the total required depth of a single layer of entanglement renormalization for a desired error is polylogarithmic in $\frac{1}{\varepsilon}$. This shows that our entanglement renormalization circuits prepare the ground state very efficiently: a circuit of depth $\mathcal{O}\left(\operatorname{polylog}\left(\frac{1}{\delta}\right)\right)$ achieves an accuracy $\delta$ on the correlation functions.

\section{The continuum limit}

The discrete wavelet transform has a natural continuum limit, in terms of continuous scaling and wavelet functions. This gives a way to interpret the continuous limit of the entanglement renormalization maps. It turns out the scaling functions are a natural UV cut-off that is compatible with the entanglement renormalization circuits, and in the critical case we find 
that we can reproduce certain conformal data exactly from a single layer of renormalization. We consider the free boson, described by bosonic fields $\phi(x), \pi(x)$ and Hamiltonian

$$
H=\frac{1}{2} \int \mathrm{d} x \pi(x)^{2}+m^{2} \phi(x)^{2}+(\partial \phi(x))^{2} .
$$

We are particularly interested in the massless case, which gives rise to a conformal field theory.

\subsection{Scaling and wavelet functions}

The continuum limit of the discrete biorthogonal wavelet transform is determined by the scaling functions. Given biorthogonal wavelet filters $g, h$ the associated scaling functions are defined in Fourier space for $a=g, h$ by

$$
\hat{\phi}^{a}(k)=\prod_{n=1}^{\infty} \frac{a_{s}\left(2^{-n} k\right)}{\sqrt{2}}
$$

and the associated wavelet functions by

$$
\hat{\psi}^{a}(k)=\frac{1}{\sqrt{2}} a_{w}\left(\frac{k}{2}\right) \hat{\phi}^{a}\left(\frac{k}{2}\right) .
$$

Both have compact support if the filters are finite, an example is shown in Fig. 4. Moreover, we can define rescaled and shifted versions

$$
\begin{aligned}
& \psi_{l, n}^{a}(x)=2^{-\frac{l}{2}} \psi^{a}\left(2^{-l} x-n\right), \\
& \phi_{l, n}^{a}(x)=2^{-\frac{l}{2}} \phi^{a}\left(2^{-l} x-n\right) .
\end{aligned}
$$

It then follows that the sets $\left\{\psi_{l, n}^{g}\right\}_{l, n \in \mathbb{Z}}$ and $\left\{\psi_{l, n}^{h}\right\}_{l, n \in \mathbb{Z}}$ form a dual basis, in the sense that

$$
\left\langle\psi_{l, n}^{g}, \psi_{l^{\prime}, n^{\prime}}^{h}\right\rangle=\delta_{l, l^{\prime}} \delta_{n, n^{\prime}}
$$

If the filters are finite and the scaling functions are square-integrable functions (which is closely related to the discrete wavelet decomposition being sufficiently stable) the sets $\left\{\psi_{l, n}^{g}\right\}_{l, n \in \mathbb{Z}}$ and $\left\{\psi_{l, n}^{h}\right\}_{l, n \in \mathbb{Z}}$ form a Riesz basis of $L^{2}(\mathbb{R})[19$.

\subsection{Entanglement renormalization for the massless boson}

One may explicitly check that if $g_{w}(k)=\left|\sin \left(\frac{k}{2}\right)\right| h_{w}(k)$ (the dispersion relation for the harmonic chain) then the scaling and wavelet functions are related as $\hat{\phi}^{g}(k)=\frac{|k|}{4\left|\sin \left(\frac{k}{2}\right)\right|} \hat{\phi}^{h}(k)$ and $\hat{\psi}^{g}(k)=\frac{|k|}{4} \hat{\psi}^{h}(k)$. Hence the wavelet functions are related precisely by the dispersion relation of the massless free boson.

If we consider correlation functions of smeared fields $\phi(f)=\int \mathrm{d} x f(x) \phi(x), \pi(f)=$ $\int \mathrm{d} x f(x) \pi(x)$ one can discretize using the scaling functions by

$$
\begin{aligned}
& \phi(f) \mapsto \sum_{n}\left\langle\phi_{l, n}^{g}, f\right\rangle q_{n} \\
& \pi(f) \mapsto \sum_{n}\left\langle\phi_{l, n}^{h}, f\right\rangle p_{n}
\end{aligned}
$$


(the inner product here is the $L^{2}(\mathbb{R})$ inner product). If the scale $l$ (which is a UV cut-off) is fine enough, on can then compute correlation functions using the (discrete) entanglement renormalization circuit to good approximation, similar to the fermionic construction described in [9]. This yields a natural way to interpret the continuous limit of bosonic entanglement renormalization as quantum field theory.

As an application, we can consider the entanglement renormalization superoperator $\Phi$, which coarse-grains operators by conjugating with a single layer of the renormalization circuit. For critical lattice models, $\Phi$ has been proposed to approximately encode the conformal data of the continuum limit of the theory [4]. For instance, for a primary field in the conformal field theory with scaling dimension $\lambda$, there should be a local operator $O$ such that $\Phi(O) \approx 2^{-\lambda} O$. We will now verify that the entanglement renormalization superoperator reproduces exactly the scaling dimensions of the $\phi$ and $\pi$ fields in the massless case, as well as the scaling dimension of a number of descendants (equal to the number of vanishing moments of the wavelet filters), similar as for the fermionic wavelet MERA [7, 9]. This is seen by considering the operators $O_{\phi}(x)=\sum_{n} \phi^{h}(x-n) q_{n}$ and $O_{\pi}(x)=\sum_{n} \phi^{g}(x-n) p_{n}$ for any $x \in \mathbb{R}$, which are the discretizations of the operators $\phi(x)$ and $\pi(x)$. It can be easily seen that the entanglement renormalization superoperator maps these operators

$$
\begin{array}{r}
O_{\phi}(x) \mapsto \sum_{n, l} \sqrt{2} h_{s}[l] \phi^{h}(x-2 n-l) q_{n} \\
=\sum_{n} \phi^{h}\left(\frac{x}{2}-n\right) q_{n}=O_{\phi}\left(\frac{x}{2}\right)
\end{array}
$$

where we use that for the scaling function Eq. $\sqrt{11}$ it holds that $\frac{1}{\sqrt{2}} \phi^{h}\left(\frac{x}{2}\right)=\sum_{n} h_{s}[n] \phi^{h}(x-$ n) 14. Similarly one finds $O_{\pi}(x) \mapsto \frac{1}{2} O_{\pi}\left(\frac{x}{2}\right)$. This corresponds, as expected, to scaling dimensions 0 and 1 . The descendants of $\phi$ correspond to $O_{L^{l} \phi}=\sum \phi^{h, l}(x-n)$ where $\hat{\phi}^{h, l}(k)=\frac{i k}{1-e^{i k}}$ for $l=1, \ldots, K$, assuming $\phi^{h}$ has $K$ vanishing moments. A vanishing moment of the scaling function $\phi^{h}$ corresponds to a factor $\left(1+e^{i k}\right)$ in the scaling filter $h_{s}$ 14. The function $\hat{\phi}^{h, l}$ is the scaling function corresponding to the filter $h_{s}(k) /\left(1+e^{i k}\right)^{l}$ and hence is such that $\partial_{x} \phi^{h}(x)=\phi^{h, 1}(x+1)-\phi^{h, 1}(x)$. However, whether $\phi^{h, l}$ is square integrable function for all $l=1, \ldots, K$ depends on the smoothness of $\phi^{h}$. If we take $x=0$ we can nevertheless define $\phi^{h, l}(n)$ for integer $n$, see Chapters 6 and 7 in [20] for technical details, so we get in any case $K$ descendants in the spectrum. In a similar fashion we obtain $K$ descendants $O_{L^{l} \pi}$ provided $\phi^{g}$ has $K$ vanishing moments.

\subsection{The massive bosonic field}

The free massive boson with mass $m$ can be approached similarly. In that case, we suppose we have two families of filters $g^{(l)}$ and $h^{(l)}$, now with $l \in \mathbb{Z}$ and such that $\sqrt{\left(m^{(l)}\right)^{2}+1} g_{w}^{(l)}(k)=$ $\sqrt{\left(m^{(l)}\right)^{2}+\sin ^{2}\left(\frac{k}{2}\right)} h_{w}^{(l)}(k)$ where $m^{(0)}=m$ and $m^{(l)}$ is the mass after $l$ layers of renormalization, as defined by Eq. (6). If these filters are chosen in a way that they converge to a fixed orthonormal filter as $l$ goes to infinity, and to a fixed pair of biorthogonal filters as in the massless case for $l$ to $-\infty$, it makes sense to define a new type of scaling and wavelet functions 
which are different at each level $l$ as a generalization of of the scaling and wavelet functions:

$$
\begin{aligned}
\hat{\phi}_{l}^{a}(k) & =\prod_{j=1}^{\infty} \frac{a^{(l+j)}\left(2^{-j} k\right)}{\sqrt{2}} \\
\hat{\psi}_{l}^{a}(k) & =\frac{1}{\sqrt{2}} a^{(l+1)}\left(\frac{k}{2}\right) \hat{\phi}_{l+1}^{a}\left(\frac{k}{2}\right)
\end{aligned}
$$

for $a=g, h$ as a generalization of of the scaling and wavelet functions defined in Eq. (11) and Eq. 12). Again, the wavelet functions $\psi_{l, n}^{a}(x)=2^{-\frac{l}{2}} \psi_{l}\left(2^{-l} x-n\right)$ for $a=g, h$ form a dual basis (provided they exist). The behaviour for $l \rightarrow \pm \infty$ is consistent with the fact that the mass term is a relevant perturbation of the conformal field theory and the theory flows from a critical massless boson to a trivial theory. As before, we can now discretize the theory using the scaling functions at some given scale and use the discrete circuit to compute correlation functions.

\subsection{Other perspectives}

The idea that wavelet theory should be a natural tool to discretize a field theory in order to perform renormalization has a long history [21]. For some recent discussions of discretizing bosonic field theories using wavelets, see [13, 22, 23. Our approach fundamentally differs from these works in that we use biorthogonal wavelets (as is natural in the bosonic setting), which moreover are specifically designed to target the Hamiltonian of the field theory (rather than using off-the-shelf wavelets such as the Daubechies wavelets). There is also a different approach to entanglement renormalization for quantum field theories, known as cMERA 24,25]. This takes a different perspective by formulating a variational class of states directly in the continuum, rather than considering a discretization. In both cases, the correlation functions of the theory are accurately reproduced up to some cut-off. The precise relation between MERA and cMERA is not very well understood, for instance it is not clear that discretizing a cMERA state could yield a MERA. Intriguingly, cMERA is formally strongly reminiscent of the continuous wavelet transform (CWT). The continuous wavelet transform [14 can be defined for a much broader class of wavelet functions $\psi$, and if $\psi$ is a biorthogonal wavelet the CWT can be discretized to a discrete wavelet transform. Reformulating cMERA as the second quantization of a CWT would therefore give a clear relationship between MERA and cMERA for free bosonic systems. However, the CWT appears to break some of the symplectic properties of the discrete biorthogonal wavelet transform and it remains an open problem to make this connection more explicit. Finally, another reason why the field theory limit of entanglement renormalization is of interest is its tentative relation to holography in theories of quantum gravity, as conjectured in [26]. The entanglement renormalization circuit can be thought of as mapping a system into one higher dimension by adding an additional 'scale' direction. An interpretation in terms of wavelets was proposed in 27] for fermions and extended to bosonic systems in 28.

\section{Conclusion}

In this work we have explained how Gaussian entanglement renormalization circuits can be naturally contructed from (and are in fact equivalent to) the second quantizations of 
biorthogonal wavelet transforms. There are a few technical aspects that deserved further study, such as a more rigorous analysis of the continuum limit, as in [9]. This poses some interesting questions. For instance, if the system is not scale invariant then our notion of wavelet functions goes beyond what is standard in the wavelet literature. The wavelet filter design problem also leaves some open questions; it would be desirable to identify sufficient conditions under which one is guaranteed to find good approximate solutions of Eq. (10).

Overall, we believe that this work, together with $[7,8]$ for the fermionic case, completes our conceptual understanding of Gaussian entanglement renormalization for free theories as the second quantization of wavelet decompositions. We hope that this offers a path towards constructing and analyzing entanglement renormalization circuits for interacting models. One clear direction is to apply perturbation theory in the wavelet basis. Another interesting direction is to investigate integrable models, where we know explicit solutions for the ground state, and try to formulate these in terms of wavelet modes.

\section{Acknowledgements}

We thank Adrián Franco Rubio for inspiring discussions during the inception of this project.

Funding information MW acknowledges funding by NWO Veni grant 680-47-459.

\section{A Construction of filters}

Next we will explain how to construct filter pairs that yield a good approximation of a given dispersion relation. Suppose we are given a dispersion relation $\omega(k)$. Let us assume that $\omega(k)=\omega(-k)$. We would like to construct a biorthogonal filter pair $(g, h)$ such that

$$
g_{w}(k) \approx \omega(k) h_{w}(k)
$$

or equivalently

$$
h_{s}(k) \approx \omega(k+\pi) g_{s}(k)
$$

We will describe a general approach to this problem inspired by the Daubechies wavelet construction, similar to the construction of Hilbert pair wavelets due to Selesnick [29] which were previously used in the construction of fermionic MERAs [8]. For this, we start with a rational approximation

$$
\omega(k+\pi) \approx \frac{a(k)}{b(k)}
$$

where $a$ and $b$ are finite symmetric sequences on $[-L, L]$. The approximation only has to be accurate around $k=0$. We will make the following ansatz for the Fourier transform of the scaling filters

$$
\begin{aligned}
& g_{s}(k)=b(k)\left(1+e^{i k}\right)^{K} f(k), \\
& h_{s}(k)=a(k)\left(1+e^{i k}\right)^{K} f(k) .
\end{aligned}
$$

The parameter $K$ determines the number of vanishing moments of the biorthogonal wavelets, just as in the Daubechies wavelet construction. By construction, $g_{s}(k)$ and $h_{s}(k)$ are small 
near $k=\pi$, and Eq. (16) is satisfied. In order for Eq. (17) to generate biorthogonal wavelet filters, they need to satisft the condition in Eq. (1) which translates to

$$
s(k) f(k) f(-k)+s(k+\pi) f(k+\pi) f(\pi-k)=2
$$

where $s(k)=a(k) b(k)\left(2 \cos \left(\frac{k}{2}\right)\right)^{2 K}$. One may try to solve this by letting $r(k)=f(k) f(-k)$. Then $r$ should be taken as a solution to the linear system

$$
\sum_{l} s[2 n-l] r[l]=\delta_{0}[n]
$$

Now, if possible, we perform a spectral factorization $r(k)=f(k) f(-k)$. A necessary and sufficient condition for this is that $r(k) \geq 0$ for all $k$. Unfortunately, we do not know of a condition on $a$ and $b$ that guarantees this. The resulting filters $(g, h)$ will have support of size $2 M$ where $M=K+2 L$. Finally, in the scale-invariant case, the stability condition that will be required in Theorem 1 can be checked explicitly for compactly supported filters by looking at the operators $P^{g}$ and $P^{h}$ defined by

$$
\left(P^{a} f\right)(k)=\left|a\left(\frac{k}{2}\right)\right|^{2} f\left(\frac{k}{2}\right)+\left|a\left(\frac{k}{2}+\pi\right)\right|^{2} f\left(\frac{k}{2}+\pi\right)
$$

on the space of polynomials of degree at most $2 M$ with zero mean. The filters yield square integrable scaling functions and uniformly bounded wavelet decomposition maps if and only if the eigenvalues of $P^{g}$ and $P^{h}$ are smaller in absolute value than 2 (see [30] or Theorem 4.2 in 31$]$ ).

For the massless harmonic chain, one particular choice for $a$ and $b$ is given by

$$
\begin{aligned}
& a(k)=\frac{1}{2}\left(e^{-i L k} d(k)^{2}+e^{i L k} d(-k)^{2}\right), \\
& b(k)=d(k) d(-k) .
\end{aligned}
$$

where $d[n]$ is a maximally flat all-pass filter with delay $\frac{1}{4}$ of degree $L[29]$, so it has the property that $e^{-i L k} d(-k) / d(k) \approx e^{-i \frac{k}{2}}$ on $k \in(-\pi, \pi)$. In Fig. 5 we show the goodness of the approximation in Eqs. (15) and (16) as a function of $K$ and $L$. The resulting filters and wavelets for $K=2, L=4$ are shown in Fig. 4. We remark that the construction in Eq. (18) is not necessarily optimal.

\section{B Construction of circuits from filters}

We now discuss how to explicitly construct a Gaussian circuit from a given pair of biorthogonal wavelet filters, and show that any translation-invariant Gaussian circuit of the form of Fig. 3 always arises from such a filter pair.

Motivated by the fermionic setting it has been extensively discussed in [32] how to construct unitary local circuits from orthogonal wavelet filters. The construction for biorthogonal wavelet filters is very similar and the symmetric case has already been discussed in [32], but for completeness we provide it here. Given a pair of biorthogonal filters $(g, h)$ of support $2 M$ we will construct a binary circuit of depth $M$ that implements the wavelet decomposition map. We will assume that $g$ and $h$ are supported on $[-M+1, M]$, which we can always achieve by 

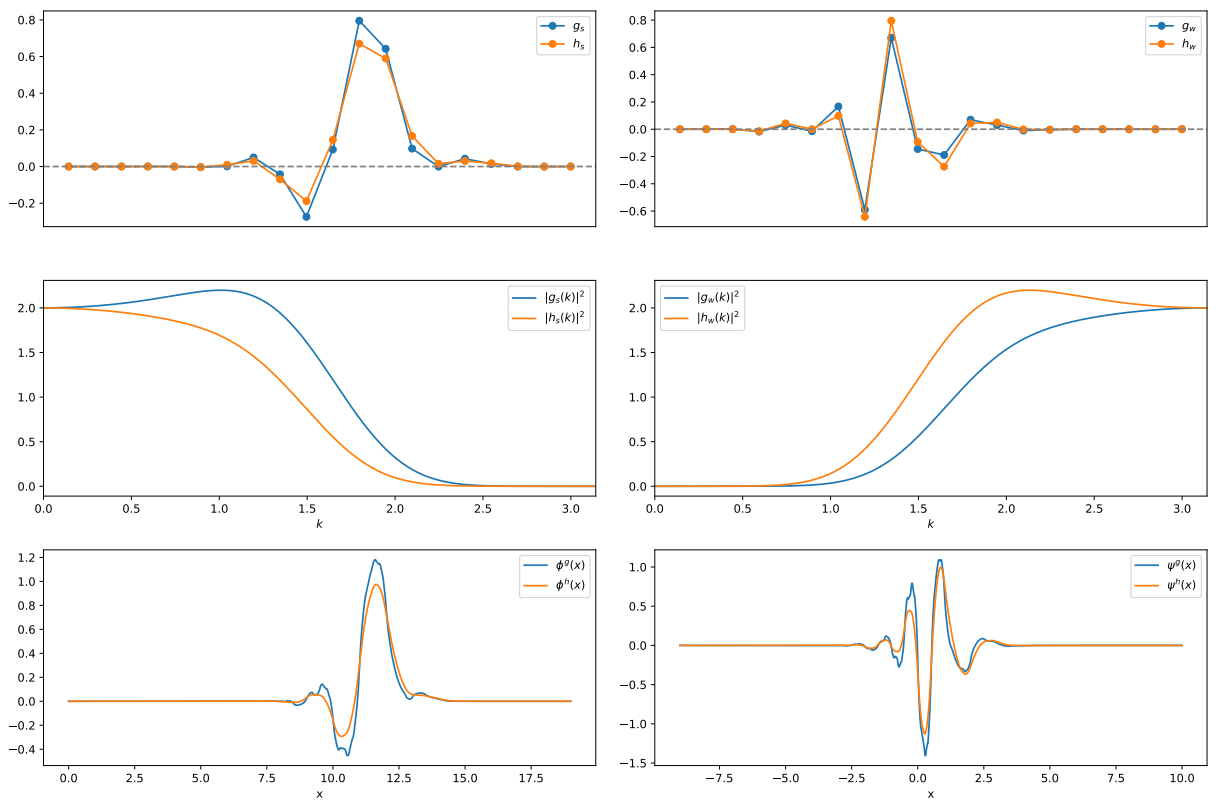

Figure 4: The filters, scaling, and wavelet functions for $K=2, L=4$ constructed using Eq. (18).

a shift. By a binary circuit of depth $M$ we mean a sequence of maps $A_{1}, \ldots A_{M}$ on $\ell^{2}(\mathbb{Z})$ such that

$$
A_{i}=\bigoplus_{n=\text { even }}\left(a_{i}\right)_{n, n+1}
$$

for $i$ even, and similarly a sum over odd terms if $i$ is odd. Here $a_{i}$ is a two by two matrix. These maps will be such that $A=A_{M} \circ \ldots \circ A_{1}$ implements the wavelet reconstruction map in the sense that $A f=W_{g}^{\top}\left(f_{\text {odd }} \oplus f_{\text {even }}\right)$ and $\left(A^{\top}\right)^{-1} f=W_{h}^{\top}\left(f_{\text {odd }} \oplus f_{\text {even }}\right)$ where $f_{\text {even }}[n]=f[2 n]$
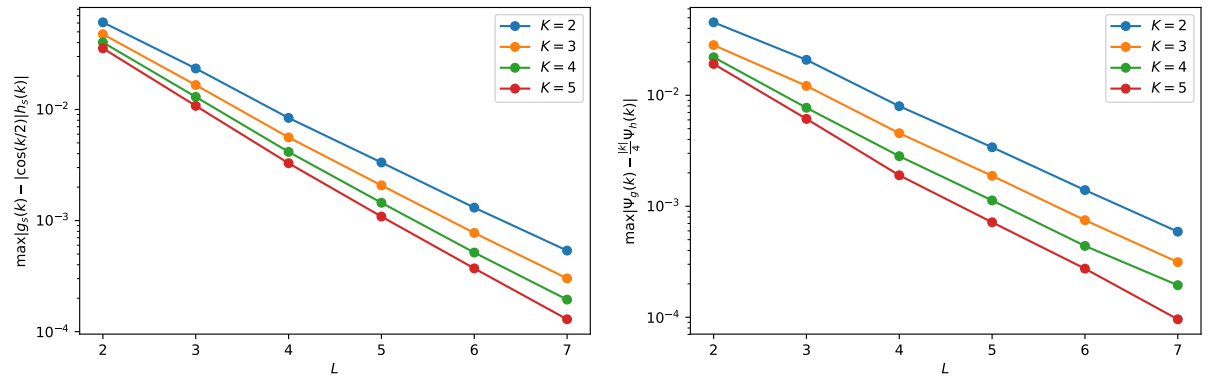

Figure 5: Approximation errors for $\varepsilon=\max _{k}\left|g_{w}(k)-\right| \sin \left(\frac{k}{2}\right)\left|h_{w}(k)\right|$ and $\max _{k}\left|\psi^{g}(k)-\frac{|k|}{4} \psi^{h}(k)\right|$ for different values of $K$ and $L$ for a filter pair constructed using Eq. (18). For fixed $K$ the error appears to decrease exponentially in $L$. 

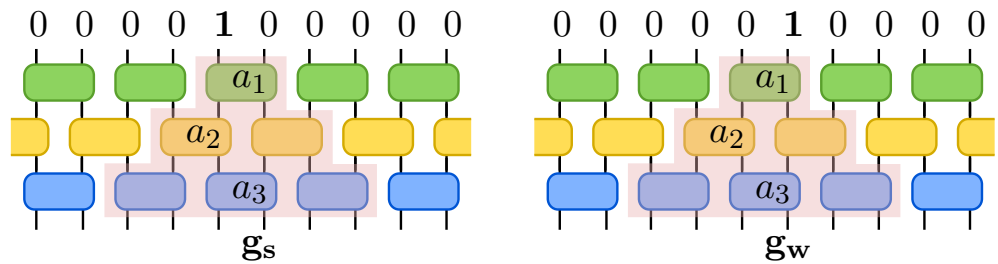

Figure 6: Illustration of Eq. (19), which gives the equations the $a_{i}$ have to satisfy in order for the circuit to implement $W_{g}$.

and $f_{\text {odd }}[n]=f[2 n-1]$, as in Fig. 3. By shift invariance this is equivalent to

$$
\begin{aligned}
A \delta_{1} & =g_{s} \\
A \delta_{2} & =g_{w} \\
\left(A^{\top}\right)^{-1} \delta_{1} & =h_{s} \\
\left(A^{\top}\right)^{-1} \delta_{2} & =h_{w}
\end{aligned}
$$

as illustrated for $g$ in Fig. 6. Now $A \oplus\left(A^{\top}\right)^{-1}$ is a binary circuit, and its second quantization gives a Gaussian bosonic quantum circuit. It remains to construct the matrices $a_{i}$ given the filters $g$ and $h$. We will need the perfect reconstruction condition Eq. (1) which can be written as

$$
\sum_{l} g_{s}[2 n+l] h_{s}[l]=\delta_{0}[n]
$$

In particular, the vectors $\left(g_{s}[-M+1], g_{s}[-M+2]\right)^{\top}$ and $\left(h_{s}[M-1], h_{s}[M]\right)^{\top}$ are orthogonal, and so are $\left(g_{s}[M-1], g_{s}[M]\right)^{\top}$ and $\left(h_{s}[-M+1], h_{s}[-M+2]\right)^{\top}$. Furthermore we will use that the wavelet filters are derived from the scaling filters as

$$
\begin{aligned}
& g_{w}[n]=(-1)^{(1-n)} h_{s}[1-n] \\
& h_{w}[n]=(-1)^{(1-n)} g_{s}[1-n] .
\end{aligned}
$$

First suppose that $M=1$. In that case we let

$$
a_{1}=\left(\begin{array}{ll}
g_{s}[0] & g_{w}[0] \\
g_{s}[1] & g_{w}[1]
\end{array}\right) .
$$

Using the perfect reconstruction condition we may now check that

$$
\left(a_{1}^{\top}\right)^{-1}=\left(\begin{array}{ll}
h_{s}[0] & h_{w}[0] \\
h_{s}[1] & h_{w}[1]
\end{array}\right)
$$

so this satisfies Eq. (19). For $M>1$ we will construct the $A_{i}$ recursively. Let

$$
\begin{array}{r}
g_{M}=\left(\begin{array}{cc}
g_{s}[M-1] & g_{s}[-M+2] \\
g_{s}[M] & g_{s}[-M+1]
\end{array}\right) \\
a_{M}=\frac{1}{\sqrt{\operatorname{det}\left(g_{M}\right)}} g_{M}
\end{array}
$$

then it is clear that $A_{M}^{-1}$ maps $g_{s}$ to a sequence $g_{s}^{(M-1)}$ on $[-M+2, M-1]$ and $A_{M}^{\top}$ maps $h_{s}$ to a sequence $h_{s}^{(M-1)}$ on $[-M+2, M-1]$ using the orthogonality properties derived from the perfect 
filter condition In the non-generic degenerate case that $\operatorname{det}\left(g_{M}\right)=0$, the size of the support can only be decreased by 1 and an additional layer is needed. Moreover, since $A_{M}$ is invariant under shifts of 2 , it is easy to see that $g_{s}^{(M-1)}$ and $h_{s}^{(M-1)}$ still satisfy the perfect reconstruction property. Finally, if we let $\alpha$ denote the map defined by $\alpha x[n]=(-1)^{(1-n)} x[1-n]$, then in order to see that $A_{M}^{-1}$ maps $g_{w}$ to the wavelet filter $g_{w}^{(M-1)}$ defined by $\alpha h_{s}^{(M-1)}$, it suffices to check that $A_{M}^{-1} \alpha=\alpha A_{M}^{\top}$ or equivalently $A_{M}^{-1}=\alpha A_{M}^{\top} \alpha$. This follows from the inversion formula for two by two matrices with determinant 1, i.e.,

$$
\left(\begin{array}{ll}
a & b \\
c & d
\end{array}\right)^{-1}=\left(\begin{array}{cc}
d & -b \\
-c & a
\end{array}\right) .
$$

Now we can recursively apply the same procedure to $\left(g^{(M-1)}, h^{(M-1)}\right)$ to construct $A_{M-1}, \ldots, A_{1}$. We have now seen that we can construct a circuit from a filter pair.

Conversely, given a circuit of the form $A=A_{M} \circ \ldots \circ A_{1}$ as described above, define filters $g$ and $h$ by Eq. (19). We can then check that these filters form perfect reconstruction filters, in the sense that $W_{h}^{\top}=W_{g}^{-1}$. If we assume $\operatorname{det}\left(a_{i}\right)=1$ for $i=1, \ldots, M$, the wavelet and scaling filters are related as in Eq. 20.

\section{Approximation theorem}

We will now state a general approximation result for translation-invariant quadratic Hamiltonians of the form Eq. (2). We obtain the theorem in the main text by specializing to the harmonic chain. Our proof strategy is inspired by the techniques in [8], with the technical complications that the wavelet transforms are not unitary and are allowed to vary layer by layer.

If $g_{s}, h_{s} \in \ell^{2}(\mathbb{Z})$ are a pair of scaling filters that satisfy the perfect reconstruction condition in Eq. (1) of the main text, then we can define corresponding wavelet filters $g_{w}, h_{w} \in \ell^{2}(\mathbb{Z})$ and single-layer decomposition maps $W_{g}, W_{h}: \ell^{2}(\mathbb{Z}) \rightarrow \ell^{2}(\mathbb{Z}) \oplus \ell^{2}(\mathbb{Z})$ such that $W_{h}^{T} W_{g}=W_{g}^{T} W_{h}=$ $\mathbb{1}$.

Now suppose that we are given a sequence of filters $g_{s}^{(l)}, h_{s}^{(l)}$ as above. Here, $l \in \mathbb{N}$ for convenience of notation. In practice, one is usually interested in a finite number of layers; in this case we may choose the sequence of filters to eventually become constant. For $a=g, h$ and $\mathcal{L} \in \mathbb{N}$, we define the $\mathcal{L}$-layer decomposition maps

$$
W_{a}^{(\mathcal{L})}: \ell^{2}(\mathbb{Z}) \rightarrow \ell^{2}(\mathbb{Z})^{\otimes(1+\mathcal{L})}, \quad W_{a}^{(\mathcal{L})}:=\left(W_{a^{(\mathcal{L}-1)}} \oplus \mathbb{1}^{\oplus(\mathcal{L}-1)}\right) \circ \ldots \circ\left(W_{a^{(1)}} \oplus \mathbb{1}\right) \circ W_{a^{(0)}},
$$

and write $W^{(\mathcal{L})}=W_{h}^{(\mathcal{L})} \oplus W_{g}^{(\mathcal{L})}$. We assume that the family is stable in the sense that the corresponding (generalized) scaling functions $\phi_{l}^{a}$ defined in Eq. (14) exist, are square integrable, and bounded in $L^{\infty}$-norm. We can also define the wavelet decomposition maps starting at layer $\mathcal{L}^{\prime} \geq 0$, that is,

$W_{a}^{\left(\mathcal{L}^{\prime}, \mathcal{L}\right)}: \ell^{2}(\mathbb{Z}) \rightarrow \ell^{2}(\mathbb{Z})^{\otimes\left(1+\mathcal{L}-\mathcal{L}^{\prime}\right)}, \quad W_{a}^{\left(\mathcal{L}^{\prime}, \mathcal{L}\right)}:=\left(W_{a}^{(\mathcal{L})} \oplus \mathbb{1}^{\oplus\left(\mathcal{L}-\mathcal{L}^{\prime}-1\right)}\right) \circ \ldots \circ\left(W_{a\left(\mathcal{L}^{\prime}+1\right)} \oplus \mathbb{1}\right) \circ W_{a}\left(\mathcal{L}^{\prime}\right)$.

Note that for $\mathcal{L}^{\prime}=0$ we recover $W_{a}^{(\mathcal{L})}$ as defined earlier. We assume that the wavelet decomposition maps are bounded. Finally, we shall assume that the filters have finite support. Then the same is true for the scaling functions. In the case that the filters are independent of 
$l$, the above notion of stability is equivalent to the familiar notion from wavelet theory. For finitely supported filters there exists an easy criterion to determine this, see [19.

For the entanglement renormalization circuit, we also insert a squeezing operation between each wavelet decomposition layer, defining $R_{a^{(l)}}, R_{a}^{(\mathcal{L})}$ and $R^{\mathcal{L}}$ for $a=g, h$ as in Eq. (9). Our approximation to the covariance matrix is then given by

$$
\begin{aligned}
\gamma_{\text {MERA }}^{q,(\mathcal{L})} & =\frac{1}{2} R_{h}^{(\mathcal{L}), \mathrm{T}} R_{h}^{(\mathcal{L})}, \\
\gamma_{\text {MERA }}^{p,(\mathcal{L})} & =\frac{1}{2} R_{g}^{(\mathcal{L}), \mathrm{T}} R_{g}^{(\mathcal{L})} .
\end{aligned}
$$

Suppose the filter pairs $g^{(l)}, h^{(l)}$ approximately satisfy the renormalized dispersion relation at each level as in Eq. (10). That is,

$$
\left|g_{w}^{(l)}(k)-\frac{\omega^{(l)}(k)}{\omega^{(l)}(\pi)} h_{w}^{(l)}(k)\right|=\left|g_{w}^{(l)}(k)-\tilde{g}_{w}^{(l)}(k)\right| \leq \varepsilon,
$$

where we have introduced the filter

$$
\tilde{g}_{w}^{(l)}(k):=\frac{\omega^{(l)}(k)}{\omega^{(l)}(\pi)} h_{w}^{(l)}(k) .
$$

This filter, together with $\tilde{h}_{w}^{(l)}(k):=\omega^{(l)}(\pi) / \omega^{(l)}(k) \times g_{w}^{(l)}(k)$, forms a pair of biorthogonal wavelet filters, with corresponding scaling filters $\tilde{g}_{s}^{(l)}, \tilde{h}_{s}^{(l)}$ that satisfy Eq. (1). However, these filters are almost never finitely supported. By construction, $\tilde{g}^{(l)}, h^{(l)}$ satisfy Eq. (3) exactly.

We now state our approximation theorem for general dispersion relations. We measure the approximation error in terms of quantities

$$
\begin{aligned}
\delta^{p} & :=\max _{n, m}\left|\gamma_{n m}^{p}-\left(\gamma_{\text {MERA }}^{p,(\mathcal{L})}\right)_{n m}\right|, \\
\delta^{q} & :=\max _{n, m}\left|\gamma_{n m}^{q}-\left(\gamma_{\text {MERA }}^{q,(\mathcal{L})}\right)_{n m}\right| .
\end{aligned}
$$

If $\omega(0)=0$, then it is also interesting to regulate the covariance matrix $\gamma^{q}$ as $\tilde{\gamma}_{n m}^{q}:=\gamma_{n m}^{q}-\gamma_{n n}^{q}$ and consider

$$
\tilde{\delta}_{n m}^{q}:=\left|\tilde{\gamma}_{n m}^{q}-\left(\tilde{\gamma}_{\text {MERA }}^{q,(\mathcal{L})}\right)_{n m}\right| .
$$

Theorem 1. Consider a translation-invariant Hamiltonian of the form of Eq. (2), with dispersion relation $\omega(k)$ such that $\omega^{(l)}(\pi) \leq 1$ and $\omega^{(l)}(k) \leq \Omega$ for $l=1, \ldots, \mathcal{L}$ for some $\Omega \geq 1$. Suppose we have a sequence of filters such that Eq. (22) holds for $\varepsilon \leq 1$, with finite support of size at most $M$ and scaling functions that are uniformly bounded by $\left\|\phi_{l}^{a}\right\|_{\infty} \leq B$ for $a=g, h$ and $l=1, \ldots, \mathcal{L}$. Assume moreover that the wavelet decomposition maps are uniformly bounded by $\left\|W_{a}^{\left(l^{\prime}, l\right)}\right\| \leq D$ for all $a=g, h, \tilde{g}$ and $1 \leq l \leq l^{\prime} \leq \mathcal{L}$, where $D \geq 1$. Then the approximation error of the covariance matrices can be bounded as follows:

$$
\begin{aligned}
\delta^{p} & \leq D^{2}\left(C 2^{-\frac{\mathcal{L}}{2}}+3 \varepsilon D \log _{2} \frac{C}{\varepsilon}\right), \\
\delta^{q} & \leq 2 D^{2}\left(C 2^{-\frac{\mathcal{L}}{2}}+3 \varepsilon D \log _{2} \frac{C}{\varepsilon}\right)\left\|\gamma^{q} \delta_{0}\right\|, \\
\tilde{\delta}_{n m}^{q} & \leq 2 D^{2}\left(C 2^{-\frac{\mathcal{L}}{2}}+3 \varepsilon D \log _{2} \frac{C}{\varepsilon}\right)\left\|\gamma^{q}\left(\delta_{n}-\delta_{m}\right)\right\|,
\end{aligned}
$$

where $C:=4 B^{2} M^{\frac{3}{2}} \Omega$. 
Our proof strategy is inspired by the techniques in [8], with the technical complications that the wavelet transforms are not unitary and are allowed to vary layer by layer. We will first bound the error that arises from only taking a finite number of layers. Let $p_{s}^{(\mathcal{L})}$ denote the projection onto the first tensor factor of $\ell^{2}(\mathbb{Z})^{\otimes(\mathcal{L}+1)}$ and $p_{w}^{(\mathcal{L})}=\mathbb{1}-p_{s}^{(\mathcal{L})}$ the projection onto the remaining tensor factors. Thus, $p_{s}^{(\mathcal{L})} W_{a}^{(\mathcal{L})} f$ is the scaling component of the decomposed signal and $p_{w}^{(\mathcal{L})} W_{a}^{(\mathcal{L})}$ its wavelet component. The following lemma confirms the intuition that, for finitely supported signals, lower-frequency wavelet modes contribute less.

Lemma 1. Suppose we have sequence of filters as above, with finite support of size at most $M$ and scaling functions that are uniformly bounded by $\left\|\phi_{l}^{a}\right\|_{\infty} \leq B$ for $a=g, h$ and $l=1, \ldots, \mathcal{L}$. Then,

$$
\left\|p_{s}^{(\mathcal{L})} W_{a}^{(\mathcal{L})} \delta_{n}\right\| \leq 2^{-\frac{\mathcal{L}-1}{2}} B^{2} M^{\frac{3}{2}}
$$

where $\delta_{n}$ is the unit signal concentrated at $n$.

Proof. Let $b$ denote the filters dual to $a$ (i.e., $b=h$ if $a=g$, and vice versa). We note that

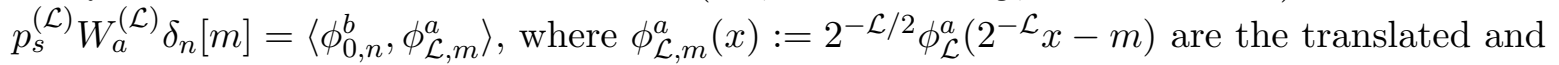
shifted scaling functions. This follows from the fact that $\left\langle\phi_{0, n}^{b}, \phi_{0, m}^{a}\right\rangle=\delta_{n m}$ and by applying inductively the fact that by definition of the scaling functions $\phi_{l+1, m}^{a}=\sum_{n} a_{s}^{(l+1)}(2 m-n) \phi_{l, n}^{a}$. Now we can proceed as in the proof of Lemma 1 in 8 and estimate

$$
\begin{aligned}
\left\|p_{s}^{(\mathcal{L})} W_{a}^{(\mathcal{L})} \delta_{n}\right\|^{2} & =\sum_{m} \mid \int_{-\infty}^{\infty} \mathrm{d} x \phi_{0}^{b}(x-n) 2^{-\frac{\mathcal{L}}{2}} \phi_{\mathcal{L}}^{a}\left(2^{\left.-\mathcal{L}_{x}-m\right)\left.\right|^{2}}\right. \\
& =\sum_{m}\left|\int_{x_{0}+n}^{x_{0}+n+M-1} \mathrm{~d} x \phi_{0}^{b}(x-n) 2^{-\frac{\mathcal{L}}{2}} \phi_{\mathcal{L}}^{a}\left(2^{-\mathcal{L}} x-m\right)\right|^{2} \\
& \leq \sum_{m}\left\|\phi_{0}^{b}\right\|^{2} \int_{x_{0}+n}^{x_{0}+n+M-1} \mathrm{~d} x\left|2^{-\frac{\mathcal{L}}{2}} \phi_{\mathcal{L}}^{a}\left(2^{-\mathcal{L}} x-m\right)\right|^{2} \\
& =2^{-\mathcal{L}}\left\|\phi_{0}^{b}\right\|^{2} \sum_{m} \int_{x_{0}+n}^{x_{0}+n+M-1} \mathrm{~d} x\left|\phi_{\mathcal{L}}^{a}\left(2^{-\mathcal{L}_{x}}-m\right)\right|^{2} \\
& \leq 2^{-\mathcal{L}+1} M^{2}\left\|\phi_{0}^{b}\right\|^{2}\left\|\phi_{\mathcal{L}}^{a}\right\|_{\infty}^{2},
\end{aligned}
$$

where in the second line we use that $\phi^{b}$ is compactly supported on $\left[x_{0}, x_{0}+M-1\right]$ for some $x_{0}$, in the third inequality we use Cauchy-Schwarz, and for the final inquality we use that at most $2 M$ terms in the sum have nonzero overlap. Finally we may estimate $\left\|\phi_{0}^{b}\right\|^{2} \leq M B^{2}$ and $\left\|\phi_{\mathcal{L}}^{a}\right\|_{\infty}^{2} \leq B^{2}$, which yields Eq. (24).

The following lemma bounds the approximation error for $\mathcal{L}$ layers as a function of an intermediate layer $\mathcal{L}^{\prime}$ that will later be chosen appropriately.

Lemma 2. Suppose we have a sequence of filters such that Eq. 22) holds, with finite support of size at most $M$ and scaling functions that are uniformly bounded by $\left\|\phi_{l}^{a}\right\|_{\infty} \leq B$ for $a=g$, $h$ and $l=1, \ldots, \mathcal{L}$. Assume moreover that the wavelet decomposition maps are uniformly bounded by $\left\|W_{a}^{\left(l^{\prime}, l\right)}\right\| \leq D$ for all $a=g, h, \tilde{g}$ and $1 \leq l \leq l^{\prime} \leq \mathcal{L}$, where $D \geq 1$. Finally, let $\mathcal{L}^{\prime} \in\{1, \ldots, \mathcal{L}\}$. Then we have the following bounds:

(i) For all $f \in \ell^{2}(\mathbb{Z})$ and $n \in \mathbb{N}$,

$$
\left|\left\langle\delta_{n}\left|\gamma^{q}-\gamma_{\text {MERA }}^{q,(\mathcal{L})}\right| f\right\rangle\right| \leq 2 D^{2}\left(\varepsilon \mathcal{L}^{\prime} D+2^{-\frac{\mathcal{L}^{\prime}-1}{2}} B^{2} M^{\frac{3}{2}} \max \left\{2\left\|\gamma^{p,(\mathcal{L})}\right\|, 1\right\}\right)\left\|\gamma^{q} f\right\| .
$$


(ii) Assuming $\omega^{(l)}(\pi) \leq 1$ for all $l=0, \ldots, \mathcal{L}-1$, we have the following bound for all $n \in \mathbb{N}$ :

$$
\left\|\left(\gamma^{p}-\gamma_{\text {MERA }}^{p,(\mathcal{L})}\right) \delta_{n}\right\| \leq D^{2}\left(\varepsilon \mathcal{L}^{\prime} D+2^{-\frac{\mathcal{L}^{\prime}-1}{2}} B^{2} M^{\frac{3}{2}} \max \left\{2\left\|\gamma^{p,\left(\mathcal{L}^{\prime}\right)}\right\|, 1\right\}\right) .
$$

Here, we recall that $\gamma^{q}(k)=\frac{1}{2 \omega(k)}$ and $\gamma^{p,(l)}(k)=\frac{1}{2} \omega^{(l)}(k)$.

Proof. (i) To prove Eq. (25), we first observe that by definition of $\tilde{g}$ it holds that

$$
R_{h^{(l)}} \omega^{(l)}(k)=\left(\omega^{(l+1)} \oplus \mathbb{1}\right) R_{\tilde{g}^{(l)}}
$$

and hence

$$
R_{h}^{(\mathcal{L})} \gamma^{p}=\left(\gamma^{p,(\mathcal{L})} \oplus \frac{1}{2} \mathbb{1}\right) R_{\tilde{g}}^{(\mathcal{L})},
$$

where $\gamma^{p,(\mathcal{L})}(k)=\frac{1}{2} \omega^{(\mathcal{L})}(k)$ denotes the covariance matrix defined using the renormalized dispersion relation. We use this, together with the fact that $4 \gamma^{p} \gamma^{q}=\mathbb{1}$ on the domain of $\gamma^{q}$ to write

$$
\begin{aligned}
\gamma^{q}-\gamma_{\text {MERA }}^{q,(\mathcal{L})} f & =\left(I-\gamma_{\text {MERA }}^{q,(\mathcal{L})} 4 \gamma^{p}\right) \gamma^{q} f \\
& =\left(W_{h}^{\left(\mathcal{L}^{\prime}\right), \mathrm{T}} W_{g}^{\left(\mathcal{L}^{\prime}\right)}-R_{h}^{(\mathcal{L}), \mathrm{T}} R_{h}^{(\mathcal{L})} 2 \gamma^{p}\right) \gamma^{q} f \\
& =\left(W_{h}^{\left(\mathcal{L}^{\prime}\right), \mathrm{T}} W_{g}^{\left(\mathcal{L}^{\prime}\right)}-R_{h}^{(\mathcal{L}), \mathrm{T}}\left(2 \gamma^{p,(\mathcal{L})} \oplus \mathbb{1}\right) R_{\tilde{g}}^{(\mathcal{L})}\right) \gamma^{q} f \\
& =\left(W_{h}^{\left(\mathcal{L}^{\prime}\right), \mathrm{T}} W_{g}^{\left(\mathcal{L}^{\prime}\right)}-W_{h}^{(\mathcal{L}), \mathrm{T}}\left(2 \gamma^{p,(\mathcal{L})} \oplus \mathbb{1}\right) W_{\tilde{g}}^{(\mathcal{L})}\right) \gamma^{q} f .
\end{aligned}
$$

for $f$ in the domain of $\gamma^{q}$. Thus,

$$
\begin{aligned}
\left|\left\langle\delta_{n}\left|\gamma^{q}-\gamma_{\text {MERA }}^{q,(\mathcal{L})}\right| f\right\rangle\right| & \leq\left|\left\langle\delta_{n}\left|W_{h}^{\left(\mathcal{L}^{\prime}\right), \mathrm{T}} p_{s}^{\left(\mathcal{L}^{\prime}\right)} W_{g}^{\left(\mathcal{L}^{\prime}\right)}\right| \gamma^{q} f\right\rangle\right| \\
& +\left|\left\langle\delta_{n}\left|W_{h}^{\left(\mathcal{L}^{\prime}\right), T} p_{w}^{\left(\mathcal{L}^{\prime}\right)}\left(W_{g}^{\left(\mathcal{L}^{\prime}\right)}-W_{\tilde{g}}^{\left(\mathcal{L}^{\prime}\right)}\right)\right| \gamma^{q} f\right\rangle\right| \\
& +\left|\left\langle\delta_{n}\left|\left(W_{h}^{(\mathcal{L}), T}\left(2 \gamma^{p,(\mathcal{L})} \oplus \mathbb{1}\right) W_{\tilde{g}}^{(\mathcal{L})}-W_{h}^{\left(\mathcal{L}^{\prime}\right), T} p_{w}^{\left(\mathcal{L}^{\prime}\right)} W_{\tilde{g}}^{\left(\mathcal{L}^{\prime}\right)}\right)\right| \gamma^{q} f\right\rangle\right| .
\end{aligned}
$$

We will bound the three terms separately, starting with the second term. By our assumption on the filters (Eq. (22)), $\left\|W_{g^{(l)}}-W_{\tilde{g}^{(l)}}\right\| \leq 2 \varepsilon$. Hence, using a telescoping sum,

$$
\left\|W_{g}^{\left(\mathcal{L}^{\prime}\right)}-W_{\tilde{g}}^{\left(\mathcal{L}^{\prime}\right)}\right\| \leq \sum_{l=0}^{\mathcal{L}^{\prime}-1}\left\|W_{g}^{\left(l+1, \mathcal{L}^{\prime}\right)}\right\|\left\|W_{g^{(l)}}-W_{\tilde{g}^{(l)}}\right\|\left\|W_{\tilde{g}}^{(l)}\right\| \leq 2 \varepsilon \mathcal{L}^{\prime} D^{2},
$$

so we obtain the estimate

$$
\left|\left\langle\delta_{n}\left|W_{h}^{\left(\mathcal{L}^{\prime}\right), T} p_{w}^{\left(\mathcal{L}^{\prime}\right)}\left(W_{g}^{\left(\mathcal{L}^{\prime}\right)}-W_{\tilde{g}}^{\left(\mathcal{L}^{\prime}\right)}\right)\right| \gamma^{q} f\right\rangle\right| \leq\left\|W_{h}^{\left(\mathcal{L}^{\prime}\right)}\right\|\left\|W_{g}^{\left(\mathcal{L}^{\prime}\right)}-W_{\tilde{g}}^{\left(\mathcal{L}^{\prime}\right)}\right\|\left\|\gamma^{q} f\right\| \leq 2 \varepsilon \mathcal{L}^{\prime} D^{3}\left\|\gamma^{q} f\right\| .
$$

The first term in Eq. (28) can be bounded directly using Lemma 1 .

$$
\left|\left\langle\delta_{n}\left|W_{h}^{\left(\mathcal{L}^{\prime}\right), T} p_{s}^{\left(\mathcal{L}^{\prime}\right)} W_{g}^{\left(\mathcal{L}^{\prime}\right)}\right| \gamma^{q} f\right\rangle\right| \leq\left\|p_{s}^{\left(\mathcal{L}^{\prime}\right)} W_{h}^{\left(\mathcal{L}^{\prime}\right)} \delta_{n}\right\|\left\|W_{g}^{\left(\mathcal{L}^{\prime}\right)} \gamma^{q} f\right\| \leq 2^{-\frac{\mathcal{L}^{\prime}-1}{2}} B^{2} M^{\frac{3}{2}} D\left\|\gamma^{q} f\right\|,
$$

and the third term may be similarly bounded as

$$
\begin{aligned}
& \left|\left\langle\delta_{n}\left|\left(W_{h}^{(\mathcal{L}), T}\left(2 \gamma^{p,(\mathcal{L})} \oplus \mathbb{1}\right) W_{\tilde{g}}^{(\mathcal{L})}-W_{h}^{\left(\mathcal{L}^{\prime}\right), T} p_{w}^{\left(\mathcal{L}^{\prime}\right)} W_{\tilde{g}}^{\left(\mathcal{L}^{\prime}\right)}\right)\right| \gamma^{q} f\right\rangle\right| \\
= & \mid\left\langle\delta _ { n } \left|\left( W_{h}^{\left(\mathcal{L}^{\prime}\right), T}\left(W_{h}^{\left(\mathcal{L}^{\prime}, \mathcal{L}\right), T} \oplus \mathbb{1}^{\oplus \mathcal{L}^{\prime}}\right)\left(2 \gamma^{p,(\mathcal{L})} \oplus \mathbb{1}^{\oplus\left(\mathcal{L}-\mathcal{L}^{\prime}\right)} \oplus 0^{\oplus \mathcal{L}^{\prime}}\right) W_{\tilde{g}}^{(\mathcal{L})}\left|\gamma^{q} f\right\rangle \mid\right.\right.\right. \\
= & \mid\left\langle\delta _ { n } \left|\left( W_{h}^{\left(\mathcal{L}^{\prime}\right), T} p_{s}^{\left(\mathcal{L}^{\prime}\right)}\left(W_{h}^{\left(\mathcal{L}^{\prime}, \mathcal{L}\right), T} \oplus 0^{\oplus \mathcal{L}^{\prime}}\right)\left(2 \gamma^{p,(\mathcal{L})} \oplus \mathbb{1}^{\oplus \mathcal{L}}\right) W_{\tilde{g}}^{(\mathcal{L})}\left|\gamma^{q} f\right\rangle \mid\right.\right.\right. \\
\leq & \left\|p_{s}^{\left(\mathcal{L}^{\prime}\right)} W_{h}^{\left(\mathcal{L}^{\prime}\right)} \delta_{n}\right\|\left\|W_{h}^{\left(\mathcal{L}^{\prime}, \mathcal{L}\right)}\right\|\left\|2 \gamma^{p,(\mathcal{L})} \oplus \mathbb{1}^{\oplus \mathcal{L}}\right\|\left\|W_{\tilde{g}}^{(\mathcal{L})}\right\|\left\|\gamma^{q} f\right\| \\
\leq & 2^{-\frac{\mathcal{L}^{\prime}-1}{2}} B^{2} M^{\frac{3}{2}} D^{2} \max \left\{2\left\|\gamma^{p,(\mathcal{L})}\right\|, 1\right\}\left\|\gamma^{q} f\right\| .
\end{aligned}
$$


By combining the three estimates we obtain Eq. (25).

(ii) To prove Eq. 26) we use Eqs. (21) and (27) to write

$$
\begin{aligned}
\gamma^{p}-\gamma_{\text {MERA }}^{p,(\mathcal{L})} & =\gamma^{p}-\frac{1}{2} R_{g}^{(\mathcal{L}), \mathrm{T}} R_{g}^{(\mathcal{L})}=\gamma^{p} R_{h}^{\left(\mathcal{L}^{\prime}\right), \mathrm{T}} R_{g}^{\left(\mathcal{L}^{\prime}\right)}-\frac{1}{2} R_{g}^{(\mathcal{L}), \mathrm{T}} R_{g}^{(\mathcal{L})} \\
& =R_{\tilde{g}}^{\left(\mathcal{L}^{\prime}\right), \mathrm{T}}\left(\gamma^{p,\left(\mathcal{L}^{\prime}\right)} \oplus \frac{1}{2} \mathbb{1}\right) R_{g}^{\left(\mathcal{L}^{\prime}\right)}-\frac{1}{2} R_{g}^{(\mathcal{L}), \mathrm{T}} R_{g}^{(\mathcal{L})}
\end{aligned}
$$

Therefore,

$$
\begin{aligned}
\left\|\left(\gamma^{p}-\gamma_{\text {MERA }}^{p,(\mathcal{L})}\right) \delta_{n}\right\| & \leq\left\|R_{\tilde{g}}^{\left(\mathcal{L}^{\prime}\right), \mathrm{T}} \gamma^{p,\left(\mathcal{L}^{\prime}\right)} p_{s}^{\left(\mathcal{L}^{\prime}\right)} R_{g}^{\left(\mathcal{L}^{\prime}\right)} \delta_{n}\right\| \\
& +\frac{1}{2}\left\|\left(R_{\tilde{g}}^{\left(\mathcal{L}^{\prime}\right), \mathrm{T}}-R_{g}^{\left(\mathcal{L}^{\prime}\right), \mathrm{T}}\right) p_{w}^{\left(\mathcal{L}^{\prime}\right)} R_{g}^{\left(\mathcal{L}^{\prime}\right)} \delta_{n}\right\| \\
& +\frac{1}{2}\left\|\left(R_{g}^{\left(\mathcal{L}^{\prime}\right), \mathrm{T}} p_{w}^{\left(\mathcal{L}^{\prime}\right)} R_{g}^{\left(\mathcal{L}^{\prime}\right)}-R_{g}^{(\mathcal{L}), \boldsymbol{T}} R_{g}^{(\mathcal{L})}\right) \delta_{n}\right\|
\end{aligned}
$$

As before we bound the three terms separately, starting with the second term. Since $\omega^{(l)}(\pi) \leq 1$, we may estimate $\left\|R_{g}^{\left(\mathcal{L}^{\prime}\right)}\right\| \leq\left\|W_{g}^{\left(\mathcal{L}^{\prime}\right)}\right\| \leq D$ and $\left\|R_{\tilde{g}}^{\left(\mathcal{L}^{\prime}\right)}-R_{g}^{\left(\mathcal{L}^{\prime}\right)}\right\| \leq 2 \varepsilon \mathcal{L}^{\prime} D^{2}$ by a telescoping sum as in Eq. (29). Thus:

$$
\frac{1}{2}\left\|\left(R_{\tilde{g}}^{\left(\mathcal{L}^{\prime}\right), \boldsymbol{T}}-R_{g}^{\left(\mathcal{L}^{\prime}\right), \boldsymbol{T}}\right) p_{w}^{\left(\mathcal{L}^{\prime}\right)} R_{g}^{\left(\mathcal{L}^{\prime}\right)} \delta_{n}\right\| \leq \frac{1}{2}\left\|\left(R_{\tilde{g}}^{\left(\mathcal{L}^{\prime}\right), \boldsymbol{T}}-R_{g}^{\left(\mathcal{L}^{\prime}\right), \boldsymbol{\top}}\right)\right\|\left\|R_{g}^{\left(\mathcal{L}^{\prime}\right)}\right\| \leq \varepsilon \mathcal{L}^{\prime} D^{3} .
$$

For the remaining terms, we note that $\omega^{(l)}(\pi) \leq 1$ also implies that $\left\|p_{s}^{\left(\mathcal{L}^{\prime}\right)} R_{g}^{\left(\mathcal{L}^{\prime}\right)} \delta_{n}\right\| \leq$ $\left\|p_{s}^{\left(\mathcal{L}^{\prime}\right)} W_{g}^{\left(\mathcal{L}^{\prime}\right)} \delta_{n}\right\| \leq 2^{-\frac{\mathcal{L}^{\prime}-1}{2}} B^{2} M^{\frac{3}{2}}$ using Lemma 1 . We can thus bound the first term by

$$
\left\|R_{\tilde{g}}^{\left(\mathcal{L}^{\prime}\right), \mathrm{T}} \gamma^{p,\left(\mathcal{L}^{\prime}\right)} p_{s}^{\left(\mathcal{L}^{\prime}\right)} R_{g}^{\left(\mathcal{L}^{\prime}\right)} \delta_{n}\right\| \leq\left\|R_{\tilde{g}}^{\left(\mathcal{L}^{\prime}\right), \mathrm{T}}\right\|\left\|\gamma^{p,\left(\mathcal{L}^{\prime}\right)}\right\|\left\|p_{s}^{\left(\mathcal{L}^{\prime}\right)} R_{g}^{\left(\mathcal{L}^{\prime}\right)} \delta_{n}\right\| \leq 2^{-\frac{\mathcal{L}^{\prime}-1}{2}} B^{2} M^{\frac{3}{2}} D\left\|\gamma^{p,\left(\mathcal{L}^{\prime}\right)}\right\|,
$$

and similarly the third term, where we find

$$
\begin{aligned}
\frac{1}{2}\left\|\left(R_{g}^{(\mathcal{L}), \boldsymbol{T}} R_{g}^{(\mathcal{L})}-R_{g}^{\left(\mathcal{L}^{\prime}\right), \boldsymbol{T}} p_{w}^{\left(\mathcal{L}^{\prime}\right)} R_{g}^{\left(\mathcal{L}^{\prime}\right)}\right) \delta_{n}\right\| & =\frac{1}{2}\left\|R_{g}^{(\mathcal{L}), \boldsymbol{T}} R_{g}^{\left(\mathcal{L}^{\prime}, \mathcal{L}\right)} p_{s}^{\left(\mathcal{L}^{\prime}\right)} R_{g}^{\left(\mathcal{L}^{\prime}\right)} \delta_{n}\right\| \\
& \leq \frac{1}{2}\left\|R_{g}^{(\mathcal{L}), \boldsymbol{T}}\right\|\left\|R_{g}^{\left(\mathcal{L}^{\prime}, \mathcal{L}\right)}\right\|\left\|p_{s}^{\left(\mathcal{L}^{\prime}\right)} R_{g}^{\left(\mathcal{L}^{\prime}\right)} \delta_{n}\right\| \\
& \leq \frac{1}{2} 2^{-\frac{\mathcal{L}^{\prime}-1}{2}} B^{2} M^{\frac{3}{2}} D^{2} .
\end{aligned}
$$

By combining the three estimates we obtain Eq. (26).

As a remark, for the critical harmonic chain we that $\omega^{(l)}(\pi)=\frac{1}{2}$, in which case it is not hard to see that the scaling of Eq. 26 can be improved to $2^{-\frac{3}{2} \mathcal{L}^{\prime}}$.

We now prove the approximation theorem stated earlier (and in Theorem 1 in the main text).

Proof of the theorem. Choosing $\mathcal{L}^{\prime}=\min \left\{\left\lfloor 2 \log _{2} \frac{C}{\varepsilon}\right\rfloor, \mathcal{L}\right\}$, we see that

$$
\begin{aligned}
\varepsilon \mathcal{L}^{\prime} D+2^{-\frac{\mathcal{L}^{\prime}-1}{2}} B^{2} M^{\frac{3}{2}} \max \left\{2\left\|\gamma^{p,\left(\mathcal{L}^{\prime}\right)}\right\|, 1\right\} & \leq \varepsilon \mathcal{L}^{\prime} D+2^{-\frac{\mathcal{L}^{\prime}-1}{2}} B^{2} M^{\frac{3}{2}} \Omega \\
& \leq 2 \varepsilon D \log _{2} \frac{C}{\varepsilon}+\max \left\{C 2^{-\frac{\mathcal{L}}{2}}, \varepsilon\right\} \\
& \leq 3 \varepsilon D \log _{2} \frac{C}{\varepsilon}+C 2^{-\frac{\mathcal{L}}{2}}
\end{aligned}
$$

where we have used that $\frac{C}{\varepsilon} \geq 2$. Now the result follows from Eq. 26, and Eq. 25) in Lemma2, choosing $f=\delta_{m}$ or $f=\delta_{m}-\delta_{n}$ in the latter (and using $\left\|\gamma^{q} \delta_{m}\right\|=\left\|\gamma^{q} \delta_{0}\right\|$ ). 
To interpret the error bounds, we note that $\left\|\gamma^{p,(\mathcal{L})}\right\|=\max _{k} \frac{\omega^{(\mathcal{L})}(k)}{2}$, which is typically $\mathcal{O}(1)$. Furthermore,

$$
\begin{aligned}
\left\|\gamma^{q} \delta_{0}\right\|^{2} & =\int_{-\pi}^{\pi} \frac{\mathrm{d} k}{\omega(k)^{2}} \\
\left\|\gamma^{q}\left(\delta_{n}-\delta_{m}\right)\right\|^{2} & =\int_{-\pi}^{\pi} \mathrm{d} k \frac{\sin ^{2}\left(\frac{1}{2}(n-m) k\right)}{\omega(k)^{2}}
\end{aligned}
$$

Finally, we observe that the theorem in the main text is just the specialization of Theorem 1 to the harmonic chain. To see this, we first normalize the dispersion relation of the harmonic chain $\omega(k)$ by a factor $\sqrt{m^{2}+1}$, so that $\omega(\pi)=1$. There $\omega^{(l)}(k) \leq 1$ and we may apply Theorem 1 with $\Omega=1$. Then,

$$
\frac{1}{m^{2}+1}\left\|\gamma^{q} \delta_{0}\right\|^{2}=\int_{-\pi}^{\pi} \frac{\mathrm{d} k}{\omega(k)^{2}}=\int_{-\pi}^{\pi} \frac{\mathrm{d} k}{m^{2}+\sin ^{2}\left(\frac{k}{2}\right)} \leq \frac{2 \pi}{m^{2}},
$$

so restoring the factor $\sqrt{m^{2}+1}$ yields the results for $\delta^{p}$ and $\delta^{q}$. In the massless case we can estimate

$$
\begin{aligned}
\left\|\gamma^{q}\left(\delta_{n}-\delta_{m}\right)\right\|^{2} & =\int_{-\pi}^{\pi} \mathrm{d} k \frac{\sin ^{2}\left(\frac{|n-m| k}{2}\right)}{\sin ^{2}\left(\frac{k}{2}\right)} \\
& \leq 2\left(\int_{0}^{\frac{2}{|n-m|}} \mathrm{d} k \frac{\pi^{2}|n-m|^{2}}{4}+\int_{\frac{2}{|n-m|}}^{\pi} \mathrm{d} k \frac{\pi^{2}}{k^{2}}\right) \\
& \leq 2 \pi^{2}|n-m|,
\end{aligned}
$$

since $\left|\sin \left(\frac{k}{2}\right)\right| \geq \frac{|k|}{\pi}$ and $\left|\sin \left(\frac{n k}{2}\right)\right| \leq \min \left\{\frac{n|k|}{2}, 1\right\}$ on the interval $(\pi, \pi)$, yielding the estimate for $\tilde{\delta}_{n m}^{q}$.

\section{References}

[1] S. R. White, Density matrix formulation for quantum renormalization groups, Physical Review Letters 69(19), 2863 (1992), doi:10.1103/PhysRevLett.69.2863.

[2] U. Schollwöck, The density-matrix renormalization group in the age of matrix product states, Annals of Physics 326(1), 96 (2011), doi 10.1016/j.aop.2010.09.012.

[3] G. Vidal, Entanglement renormalization, Physical Review Letters 99, 220405 (2007), doi:10.1103/PhysRevLett.99.220405.

[4] G. Evenbly and G. Vidal, Quantum criticality with the multi-scale entanglement renormalization ansatz, In Strongly Correlated Systems, pp. 99-130. Springer (2013).

[5] I. H. Kim and B. Swingle, Robust entanglement renormalization on a noisy quantum computer (2017), arXiv:1711.07500.

[6] J. Preskill, Quantum computing in the nisq era and beyond, Quantum 2, 79 (2018), doi:10.22331/q-2018-08-06-79.

[7] G. Evenbly and S. R. White, Entanglement renormalization and wavelets, Physical Review Letters 116, 140403 (2016), doi:10.1103/PhysRevLett.116.140403. 
[8] J. Haegeman, B. Swingle, M. Walter, J. Cotler, G. Evenbly and V. B. Scholz, Rigorous free-fermion entanglement renormalization from wavelet theory, Physical Review X 8 , 011003 (2018), doi:10.1103/PhysRevX.8.011003.

[9] F. Witteveen, V. Scholz, B. Swingle and M. Walter, Quantum circuit approximations and entanglement renormalization for the Dirac field in 1+1 dimensions (2019), arXiv: 1905.08821.

[10] G. Evenbly and G. Vidal, Entanglement renormalization in free bosonic systems: realspace versus momentum-space renormalization group transforms, New J. Phys. 12, 025007 (2010), doi:10.1088/1367-2630/12/2/025007.

[11] E. Knill, R. Laflamme and G. J. Milburn, A scheme for efficient quantum computation with linear optics, nature 409(6816), 46 (2001), doi:10.1038/35051009.

[12] K. Marshall, R. Pooser, G. Siopsis and C. Weedbrook, Quantum simulation of quantum field theory using continuous variables, Physical Review A 92(6), 063825 (2015), doi:10.1103/PhysRevA.92.063825.

[13] G. K. Brennen, P. Rohde, B. C. Sanders and S. Singh, Multiscale quantum simulation of quantum field theory using wavelets, Physical Review A 92(3), 032315 (2015), doi:10.1103/PhysRevA.92.032315.

[14] S. Mallat, A Wavelet Tour of Signal Processing, Academic Press (2008).

[15] K. Audenaert, J. Eisert, M. B. Plenio and R. F. Werner, Entanglement properties of the harmonic chain, Physical Review A 66(4), 042327 (2002), doi:10.1103/PhysRevA.66.042327.

[16] M. B. Plenio, J. Eisert, J. Dreissig and M. Cramer, Entropy, entanglement, and area: analytical results for harmonic lattice systems, Physical Review Letters 94(6), 060503 (2005), doi:10.1103/PhysRevLett.94.060503.

[17] D. F. Walls and G. J. Milburn, Quantum optics, Springer Science \& Business Media (2007).

[18] J. Borregaard, M. Christandl and D. Stilck França, Noise-robust exploration of quantum matter on near-term quantum devices (2019), arXiv:1909.04786.

[19] A. Cohen, I. Daubechies and J.-C. Feauveau, Biorthogonal bases of compactly supported wavelets, Communications on Pure and Applied Mathematics 45(5), 485 (1992), doi:10.1002/cpa.3160450502.

[20] G. Strang and T. Nguyen, Wavelets and filter banks, SIAM (1996).

[21] G. Battle, Wavelets and renormalization, World Scientific (1999).

[22] F. Bulut and W. N. Polyzou, Wavelets in field theory, Physical Review D 87(11), 116011 (2013), doi:10.1103/PhysRevD.87.116011.

[23] A. Stottmeister, V. Morinelli, G. Morsella and Y. Tanimoto, Operator-algebraic renormalization and wavelets (2020), arXiv:2002.01442. 
[24] J. Haegeman, T. J. Osborne, H. Verschelde and F. Verstraete, Entanglement renormalization for quantum fields in real space, Physical Review Letters 110, 100402 (2013), doi:10.1103/PhysRevLett.110.100402.

[25] A. Franco-Rubio and G. Vidal, Entanglement and correlations in the continuous multiscale entanglement renormalization ansatz, Journal of High Energy Physics 2017(12), 129 (2017), doi:10.1007/JHEP12(2017)129.

[26] B. Swingle, Entanglement renormalization and holography, Physical Review D 86, 065007 (2012), doi:10.1103/PhysRevD.86.065007.

[27] X.-L. Qi, Exact holographic mapping and emergent space-time geometry (2013), arXiv: 1309.6282 .

[28] S. Singh and G. K. Brennen, Holographic construction of quantum field theory using wavelets (2016), arXiv:1606.05068.

[29] I. W. Selesnick, The design of approximate Hilbert transform pairs of wavelet bases, IEEE Transactions on Signal Processing 50(5), 1144 (2002), doi:10.1109/78.995070.

[30] A. Cohen and I. Daubechies, A stability criterion for biorthogonal wavelet bases and their related subband coding scheme, Duke Mathematical Journal 68(2), 313 (1992), doi:10.1215/S0012-7094-92-06814-1.

[31] A. Cohen and R. D. Ryan, Wavelets and multiscale signal processing, Springer (1995).

[32] G. Evenbly and S. R. White, Representation and design of wavelets using unitary circuits, Physical Review A 97(5), 052314 (2018), doi:10.1103/PhysRevA.97.052314. 